\title{
MicroRNA-141 Targets Sirt1 and Inhibits Autophagy to Reduce HBV Replication
}

\author{
Ying Yang ${ }^{a}$ Yanning Liu ${ }^{a}$ Jihua Xue ${ }^{b}$ Zhenggang Yang ${ }^{a}$ Yu Shi ${ }^{a}$ Yixian Shia \\ Guohua Lou ${ }^{\mathrm{a}}$ Shanshan Wua Jinjin Qia Weixia Liu ${ }^{\mathrm{a}}$ Jing Wang ${ }^{\mathrm{a}}$ Zhi Chen $^{\mathrm{a}}$
}

aState Key Laboratory for Diagnosis and Treatment of Infectious Diseases, The First Affiliated Hospital, School of Medicine, Zhejiang University. Collaborative Innovation Center for Diagnosis and Treatment of Infectious Diseases, Hangzhou, 'Department of Infectious Diseases, The First Affiliated Hospital of Anhui Medical University, Hefei, China

\section{Key Words}

$\mathrm{HBV} \cdot$ Autophagy $・$ Sirt1 $\cdot \mathrm{miR}-141$

\begin{abstract}
Background/Aims: About 400 million individuals are chronically infected with hepatitis B virus, at high risk of developing liver cirrhosis and hepatocellular carcinoma. Recent studies have demonstrated an interaction between hepatitis B virus replication and autophagy activity of hepatocytes. In the present study, we aimed to investigate the role of miR-141 in regulating autophagy and hepatitis B virus replication. Methods: The expression of HBVDNA, miR-141 and Sirt1 mRNA was determined by quantitative real-time PCR analysis. The expression of HBsAg and HBeAg was determined by ELISA. Western blotting was performed to detect protein expression. The LC3 puncta was determined by immunofluorescence. To test whether miR-141 directly regulate the expression level of Sirt1 mRNA, dual-luciferase reporter gene assay was performed. Results: In vitro studies showed that miR-141 mimic inhibited the autophagic response, hepatitis B virus and the expression of Sirt1 in hepatocytes. And transfection with miR-141 inhibitor enhanced autophagic response and Sirt1 expression. The autophagy induced by overexpression of Sirt 1 was inhibited by miR-141 mimic. In addition, miR-141 mimic also decreased the expression of Sirt1 mRNA. Sirt1 was predicted as a potential miR-141 target by bioinformatic analysis of its 3'-UTR, and confirmed by luciferase reporter assays which analyzing the interaction of miR-141 with the wild-type or the mutated Sirt1 3'-UTR. Conclusion: We have therefore demonstrated a role of miR-141 in regulating autophagy-mediated hepatitis B virus inhibition by targeting Sirt1, and may provide potential targets for drug development.
\end{abstract}

\section{Introduction}

Hepatitis B virus (HBV) belongs to the family Hepadnavirus [1]. HBV causes 400 million chronic HBV infection (CHB), which is correlated with the development of liver cirrhosis and hepatocellular carcinoma (HCC) [2-4]. However, the current two types of approved antiviral 


\section{Cellular Physiology Cell Physiol Biochem 2017;41:310-322 \begin{tabular}{ll|l} 
DOI: 10.1159/000456162 & O 2017 The Author(s). Published by S. Karger AG, Basel \\
www.karger.com/cpb
\end{tabular} \\ Yang et al.: Inhibition of HBV Replication by MicroRNA-141-Sirt1-Autophagy}

therapies cannot completely prevent liver diseases, only a limited number of patients achieve HBsAg loss, which is considered a "functional cure". During HBV infection, host cellular components and machinery must be utilized to complete replication and mediate pathogenesis [5]. However, the details of the host-virus interactions such as autophagy worth to be further clarified. To investigate the complex network of interactions with the host in the course of HBV infection is helpful to define new targets for antiviral strategies [6].

Autophagy, an evolutionarily conserved intracellular process, engulfs long-lived proteins and damaged organelles for degradation and recycling [5]. The intracellular process of autophagy has been exploited by HBV $[7,8]$. Sirt1, the most widely studied sirtuin, plays an important role in regulating the autophagy by deacetylation of autophagy-related proteins [9]. In addition, it has been shown that Sirt1 may facilitate HBV replication in hepatocytes, and target proteins implicated in HBV core promoter transcriptional regulation, especially PGC- $1 \alpha$ and FXR $[10,11]$.

miRNAs are a class of highly conserved small non-coding RNAs, which are key regulators of gene expression by targeting mRNAs, leading to translation repression or degradation [12]. It has been reported that miRNAs are involved in autophagy regulation [13]. In addition, studies show miR-141 inhibits various kinds of virus infection, such as HBV. Therefore, we hypothesized whether hsa-miR-141 inhibits HBV through inhibition of autophagy mediated by Sirt1.

The present study demonstrated the potential role of miR-141 in modulating autophagy. Our findings demonstrated overexpression of miR-141 inhibited autophagy, thus facilitating the inhibition of HBV. Further, Sirt1 was identified as a novel target of miR-141 in HBV inhibition. These findings provide a novel perspective in the host-virus relationship in HBV infection.

\section{Materials and Methods}

\section{Antibodies and reagents}

The following antibodies or reagents were used: Sirt1 (Abcam, Cambridge, MA, USA); antibody against p62 (MBL International, Nagoya, Japan) ; antibody to LC3 (Sigma, Spruce, St. Louis, USA) and antibody to $\beta$-actin (Santa Cruz, Dallas, TX, USA); Alexa Fluor 488-conjugated secondary Antibody (Molecular Probes, Junction, OR, USA), DAPI (Molecular Probes, Junction, OR, USA), Rapamycin (Rap, Sigma, Spruce, St. Louis, USA), 3-methyladenine (3-MA) and chloroquine (CQ) (Sigma, Spruce, St. Louis, USA). Rap (100 nM) exposure was for $24 \mathrm{~h}$, and Rap (50 $\mathrm{nM}$ ) exposure was for $48 \mathrm{~h}$ used as a positive control. In terms of the autophagic flux, $5 \mathrm{mM} \mathrm{3-MA}$ and $20 \mu \mathrm{M}$ CQ was added to the medium $12 \mathrm{~h}$ before cell harvest. In other aspects, 10 mM 3-MA was added to the medium $2 \mathrm{~h}$ before cell harvest. miR-141 mimic (20nM) or miR-141 inhibitor (20nM) or miR-141 mimic negative control (MMNC, 20nM) or miR-141 inhibitor negative control (MINC, 20nM) were from Ribobio (Guangzhou, China).

\section{Plasmids}

pEGFP-LC3 [14] and pSirt1 [15] were described previously. According to standard procedures, as described, the plasmid with 1.3mer HBV genomic DNA (GeneBank:U95551.1) (pCDNA3.1-HBV1.3, pHBV1.3) was constructed in our laboratory [16]. pCDNA3.1 was used as the vector. pGV306-Sirt1 and pCON172 was constructed from Genechem, Inc. (Shanghai, China). pGV306-Sirt1 (pSirt1-widetype, pSirt1WT) was mutated to construct the mutated plasmid containing the mutated Sirt1 3'-UTR (pSirt1-mutation, pSirt1-Mut). It was conducted with the QuikChange Site-Directed Mutagenesis Kit from Stratagene (Santa Clara, CA, USA) according to the manufacturer's protocol. The pSirt1-Mut plasmid was sequenced in Sangon Biotech (Shanghai) Co., Ltd.

\section{Cell culture and transient transfection}

HeLa/GFP-LC3 cells is a stable cell line that expresses the fusion protein of green fluorescence protein (GFP) and LC3. HepG2.2.15 is HepG2-derived cell line that hosts the HBV genome and produces HBV particles. HeLa/GFP-LC3 cells, HepG2.2.15 and Huh7 cells were obtained from State Key Laboratory for 


\section{Cellular Physiology Cell Physiol Biochem 2017;41:310-322

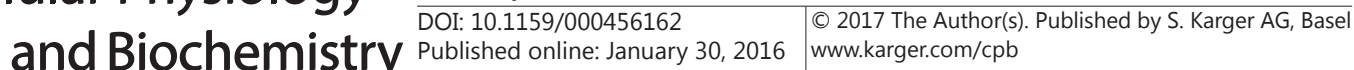 \\ Yang et al.: Inhibition of HBV Replication by MicroRNA-141-Sirt1-Autophagy}

Diagnosis and Treatment of Infectious Diseases, The First Affiliated Hospital, School of Medicine, Zhejiang University. These cells were cultured in Dulbecco's Modified Eagle Medium (DMEM, GIBCO, Grand Island, NY, USA) containing 10\% fetal bovine serum (FBS, Hyclone, Logan, UT, USA) and maintained at $37^{\circ} \mathrm{C}$ in a humidified $5 \% \mathrm{CO}_{2}$ atmosphere. Plasmids $(0.5 \mu \mathrm{g}$ in 24 -well culture plate or $2 \mu \mathrm{g}$ in 6 -well culture plate) and miRNAs $(20 \mathrm{nM})$ were co-transfected or miRNAs $(20 \mathrm{nM})$ were transfected into cells at the indicated concentrations using Lipofectamine 2000 (Invitrogen, Carlsbad, CA, USA) $24 \mathrm{~h}$ after plating following the manufacturer's protocol [17].

\section{HBV replication analysis}

Cell culture media was collected at $48 \mathrm{~h}$ post-transfection and centrifugated to remove debris before analysis. Supernatant HBsAg and HBeAg levels were determined using ELISA kits (Architect HBsAg, Abbott, Abbott Park, IL; AXSYM HBeAg, Abbott, Abbott Park, IL, USA) [18]. HBV DNA was extracted and quantified by real-time qPCR using the kit (Acon, Hangzhou, China) following the manufacturer's protocol.

\section{Immunofluorescence}

Cells were grown on glass coverslips in 24-well plates as described previously [19]. For GFP-LC3 dots, cells were fixed with $4 \%$ paraformaldehyde followed by permeabilization with $0.3 \%$ Triton X-100. For LC3 staining, after cells were fixed and permeabilized, cells were incubated with primary LC3 antibody and then Alexa Fluor 488-conjugated secondary Antibody. The cells were fixed in 4\% paraformaldehyde for $15 \mathrm{~min}$ and viewed by confocal microscopy (Olympus Inc., Center Valley, PA, USA). The exposure time for immunofluorescent detection is $1 / 2.2 \mathrm{~s}$. The nucleus was stained with DAPI if necessary. Approximately 50 cells in each group were counted. The percentage of cells with GFP-LC3 or LC3 was determined using the Image-Pro Plus 5.0 software (Media Cybernetics, Inc., Bethesda, MD, USA).

\section{Western-blot analysis}

Cells were washed with ice-cod PBS, and the cell lysates were prepared using RIPA lysis buffer containing a protease inhibitor PMSF. The protein content of different fractions was determined by a BCA method (Thermo Scientific , Grand Island, NY, USA). Equivalent amounts of protein $(20 \mu \mathrm{g})$ were separated by $12 \%$ SDS-PAGE and transferred to a polyvinylidene difluoride membrane (PVDF, Millipore, Billerica, MA, USA). The membrane was blocked in 5\% nonfat milk in TBST and incubated with primary Antibodies at $4^{\circ} \mathrm{C}$ overnight. After washing, the membrane was then incubated with horseradish peroxidase (HRP)conjugated secondary antibodies (Southern Biotechnology Associates, Inc., Birmingham, AL, USA) for 1h at room temperature and visualized with enhanced ECL (Thermo Scientific, Grand Island, NY, USA) following exposure to X-ray films [20]. The relative band intensity was quantified by using the software Image J (NIH, Bethesda, MD, USA) [21].

\section{Real-time qRT-PCR analysis}

RNA was extracted with mirVana ${ }^{\mathrm{TM}}$ miRNA Isolation Kit (AM1561, Ambion, Grand Island, NY, USA). For miRNA, the expression levels of hsa-miR-141-3p was detected using Takara kit (PrimeScript ${ }^{\mathrm{TM}}$ RT reagent Kit with gDNA Eraser, SYBR Premix EX Taq ${ }^{\mathrm{TM}}$ II) with primers Bulge-LoopTM miRNA qRT-PCR Primer Set (Ribobio, Guangzhou, China), and normalized to U6 with primers U6 snRNA qRT-PCR Primer Set (Ribobio, Guangzhou, China). For mRNA, the first-strand cDNA synthesis was performed using Kit (Takara, Dalian, China), then the expression of Sirt1 was assessed by PCR amplification. The primer sequences (Sangon, Shanghai, China) used for PCR were: Sirt1 (NM_012238.4), 5'- GCTGGCCTAATAGAGTGGCAA -3' (forward), 5'- CTCAGCGCCATGGAAAATG -3' (reverse); GAPDH (NM_008084.2), 5'-3' CCATGTTCGTCATGGGTGTGAACCA (forward); 5'-3' GCCAGTAGAGGCAGGGATGATGTTC (reverse). The PCR amplification was performed using the Applied Biosystems 7900 Sequence Detection system (Applied Biosystems, Foster City, CA, USA). The miR-141 and mRNA (Sirt1 and GAPDH) expression was calculated using $\Delta \mathrm{Ct}=\left(\mathrm{Ct}_{\mathrm{miR}-141}-\mathrm{Ct}_{\mathrm{U} 6}\right)$ and $\Delta \mathrm{Ct}=$ $\left(\mathrm{Ct}_{\mathrm{Sirt1}}-\mathrm{Ct}_{\mathrm{GAPDH}}\right)$ respectively. The relative expression of miR-141 was calculated using the $2^{-\Delta \Delta \mathrm{Ct}}$ method $\left(\Delta \Delta \mathrm{Ct}=\Delta \mathrm{Ct}{ }_{\text {experiment }}-\Delta \mathrm{Ct}{ }_{\text {control }}\right)[22]$.

\section{Luciferase reporter assays}

HepG2.2.15 cells were plated in 24-well plates $24 \mathrm{~h}$ prior to transfection. pGV306 luciferase reporter plasmids containing either a wide-type or mutated Sirt1 3'UTR were co-transfected with miR-141 mimic or 


\section{Cellular Physiology Cell Physiol Biochem 2017;41:310-322

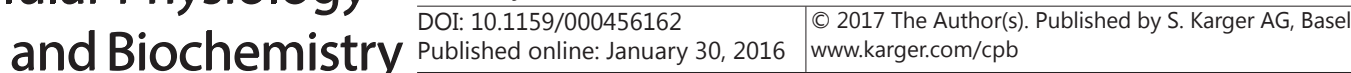 \\ Yang et al.: Inhibition of HBV Replication by MicroRNA-141-Sirt1-Autophagy}

MMNC using Lipofectamine 2000 (Invitrogen, Carlsbad, CA, USA). Cells were harvested 48h later, the Dual Luciferase Assay System from Promega was used to measure the luciferase reporter activities [23].

Statistical analysis

Independent T-test was to evaluate the significance of the results between groups. The data were analyzed by SPSS 17.0 for Windows (SPSS Inc, Chicago, IL, USA). The data were represented as mean \pm SEM. ${ }^{*} P<0.05$ and ${ }^{* *} P<0.01$ was considered statistically significant.

\section{Results}

miR-141 inhibits HBV expression and replication

miR-141 was examined to confirm its inhibition of HBV by co-transfection of Huh7 with HBV1.3 and miR-141 mimic or transfecting miR-141 mimics into HepG2.2.15 cells. As shown in Fig. 1(a-f), compared to the Mock and MMNC groups, the percentages of HBsAg/HBeAg expression levels and viral DNA were significantly reduced. These data validated that miR141 could repress HBV.

\section{miR-141 inhibits autophagosome formation}

It has been reported that autophagic vacuoles were induced by HBV [24]. We first evaluated the autophagic activity affected by miR-141 to determine the percentage of cells with LC3 puncta.

By using the PI3KC3 inhibitor 3-MA, the enzyme essential for the formation of autophagosomes was inhibited, reducing the autophagy activity. As shown in Fig. 2a and $2 \mathrm{~b}$, it is bright and punctate in the presence of HBV (in Mock and MMNC groups), but in the presence of miR-141 mimic or 3-MA, the GFP-LC3 signal is weak.

(a)

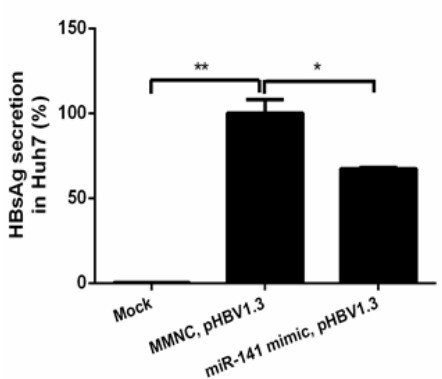

(d)

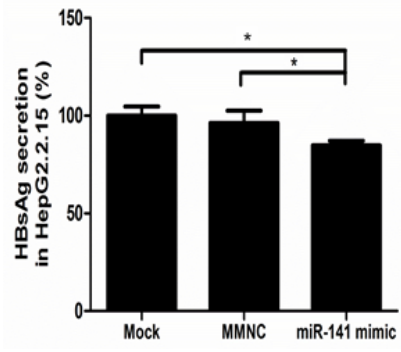

(b)

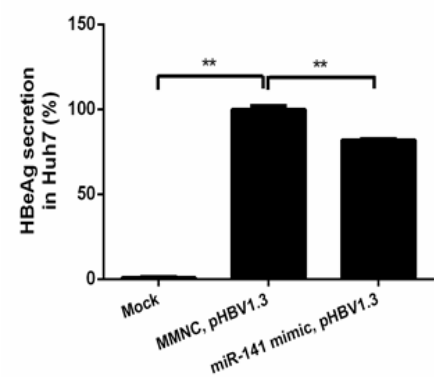

(e)

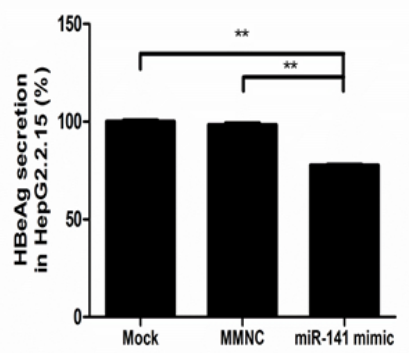

(c)

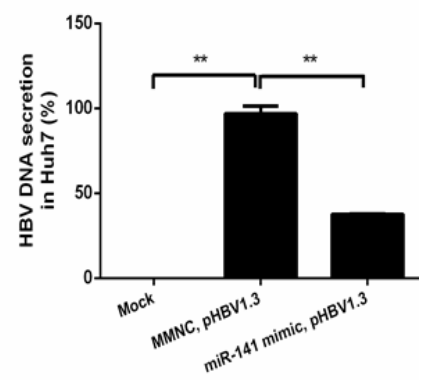

(f)

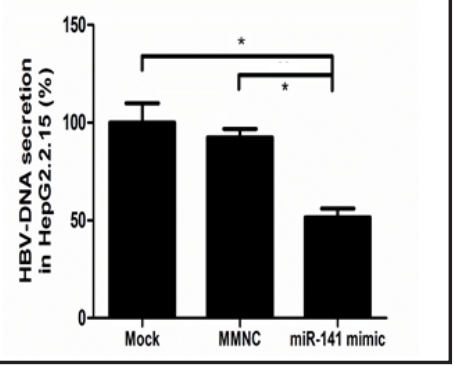

Fig. 1. miR-141 mimic inhibits HBV replication. Co-transfection of Huh7 with HBV1.3 and miR-141 mimic, transfection of HepG2.2.15 cells with miR-141 mimic represses HBV protein expression and HBV DNA replication. miR-141 mimic negative control (MMNC) and transfection reagent groups (Mock) were used as negative controls. The culture supernatant $48 \mathrm{~h}$ after transfection with miR-141 mimic was harvested. HBsAg (a, d) and HBeAg (b, e) of was determined by ELISA assay, and HBV DNA(c, f) was detected by qPCR. The results represent the mean data from three independent experiments. ${ }^{*}, P<0.05,{ }^{*}, P<0.01$. 
(a)

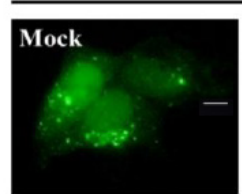

\section{MMNC}

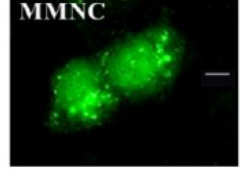

(c)
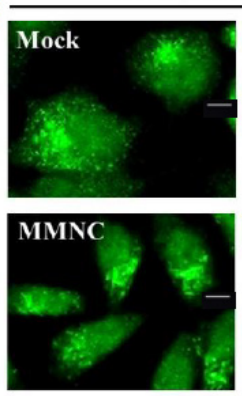
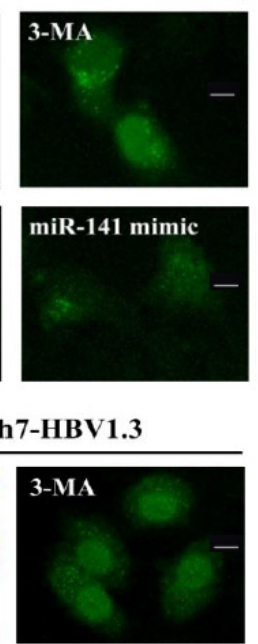

miR-141 mimic (b)

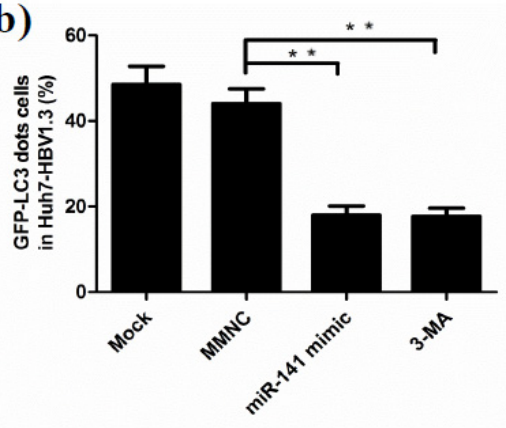

(d)

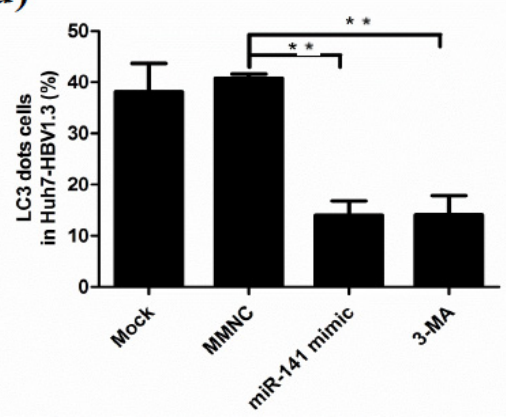

Fig. 2. miR-141 mimic inhibits autophagosome formation. Huh7 cells were transfected with pEGFP-LC3, and after $12 \mathrm{~h}$ they were co-transfected with miR-141 mimic or miR-141 mimic negative control (MMNC) with the pHBV1.3 vector. After transfection for 48h, the pEGFP-LC3 dots were visualized with a confocal fluorescence microscope. The representative images are shown above (a). (b) Determination of the frequency of pHBV1.3-transfected cells (Huh7-HBV1.3) displaying pEGFP-LC3 dots was performed as described in Materials and Methods ( $\mathrm{n}=50$ cells). (c) Huh7 cells were co-transfected with miR-141 mimic or MMNC with the pHBV1.3 vector. After transfection for $48 \mathrm{~h}$, the LC3 dots were visualized with a confocal fluorescence microscope. The representative images are shown above. (d) Percentage of Huh7-HBV1.3 cells displaying LC3 dots was determined. The data were analyzed ( $\mathrm{n}=50$ cells). Scale size: $10 \mu \mathrm{m}$. All the results represent the mean \pm SEM data from three independent experiments. ${ }^{*}, P<0.01$.

To further confirm that miR-141 inhibits autophagosomes, we conducted immunofluorescence with primary LC3 antibody to observe LC3 dots. As shown in Fig. 2c and 2d, it is bright and punctate in the presence of HBV (in Mock and MMNC groups), but in the presence of miR-141 mimic or 3-MA the LC3 dot signal is weak. These data together illustrated that miR-141 inhibits the autophagic response induced by HBV1.3.

\section{miR-141 simultaneously reduces the expression of Sirt1 and autophagy activity}

Western blot results (Fig. 3) showed that transient transfection with miR-141 mimic reduced the expression levels of Sirt1, while miR-141 inhibitor increased the amount of Sirt1 in Huh7-HBV1.3. Meanwhile, transient transfection with miR-141 mimic reduced the amount of LC3-II and the increased amount of p62, suggesting the inhibited autophagy in Huh7HBV1.3. In contrast, transient transfection with miR-141 inhibitor activated autophagy in Huh7-HBV1.3, as suggested by the increased amount of LC3-II and the reduced amount of p62. Our results showed that miR-141 might not only regulate autophagy activity, but also affected the expression of Sirt1. Now we propose the hypothesis that miR-141 might regulate autophagy activity mediated by Sirt1.

Sirt1 is a target of miR-141

TargetScan (www.targetscan.org), as a target gene prediction program, revealed that Sirt1 might be a candidate miR-141 gene target. Sequence analysis revealed that there was a 
(a)

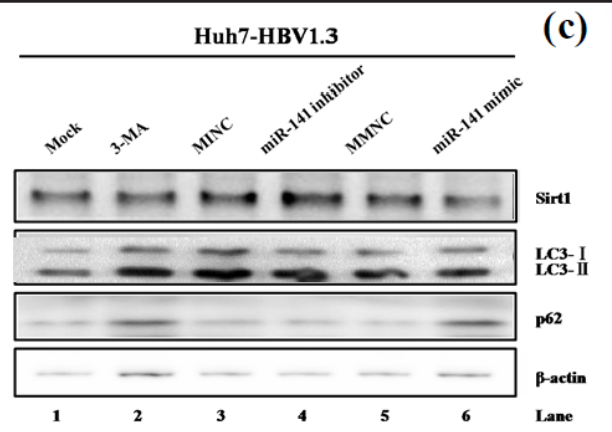

(b)

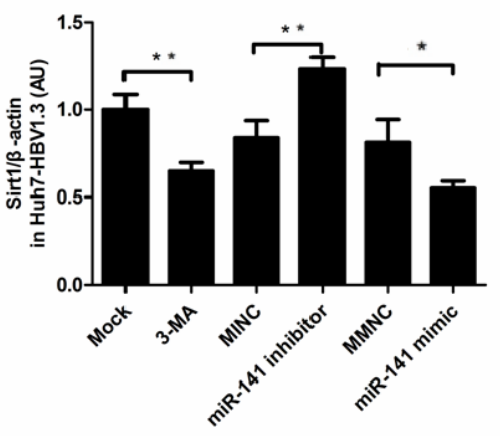

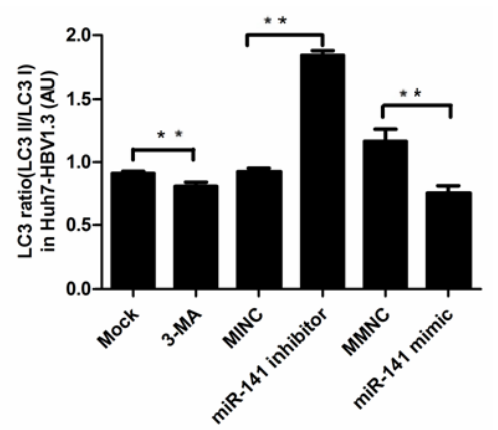

(d)

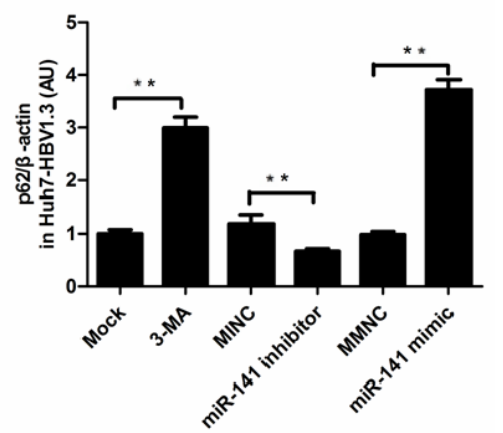

Fig. 3. The autophagic activity and the amount of Sirt1were simultaneously decreased by miR-141 in Huh7 co-transfected with HBV1.3. Huh7 cells were co-transfected with miR-141 mimic or miR-141 inhibitor with the pHBV1.3 vector. After transfection for 46h, pHBV1.3-transfected cells (Huh7-HBV1.3) were left untreated or were treated with 10mM 3-MA for 2h. Cells co-transfected with the pHBV1.3 vector and miR-141 mimic negative control (MMNC, 20nM) or miR-141 inhibitor negative control (MINC, 20nM) or transfection reagent (Mock) was used as a negative control. The Sirt1, LC3, and p62 levels were detected by Western blot, and the representative images were shown above (a). The ratios of Sirt1/ $\beta$-actin (b), LC3-II/LC3-I (c) and p62/ $\beta$-actin (d) were shown above. All the results represent the mean \pm SEM data from three independent experiments. ${ }^{*}, P<0.05,{ }^{* *}, P<0.01$.

candidate miR-141 binding site in the Sirt1 mRNA 3'-untranslated region (UTRs) (Fig. 4a). The 3'UTR containing the binding site sequence (160bp) was cloned into pGV306 to construct the reporter plasmid pGV306-Sirt1 (Fig. 5a). pGV306-Sirt1 vector was co-transfected with miR141 mimic into HepG2.2.15. Luciferase assay results demonstrated that miR-141 reduced the luciferase activity of pGV306-Sirt1 significantly (Fig. 5b). TargetScan database showed the seed sequence between hsa-miR-141-3p and the Sirt1 3'UTR (Fig. 4b). This seed sequence is highly conserved among many kinds of species (Fig. 4c). Next, Luc reporter containing the seed sequence mutant was constructed, which was called pSirt1-Mut. HepG2.2.15 cells were co-transfected pSirt1-Mut or pSirt1-WT with or without miR-141, then the luciferase reporter activity was assayed. As shown in Fig. 5c, miR-141 mimic did not change reporter activity in cells transfected with pSirt1-Mut, while miR-141 mimic significantly reduced reporter activity in cells transfected with pSirt1-WT. These results above indicated that miR141 regulated Sirt1 expression by targeting Sirt1 3'-UTR binding site.

\section{miR-141 regulates the expression of Sirt 1 mRNA}

In addition to miR-141 regulation for the expression levels of Sirt1 protein, we further determined the amount of Sirt1 mRNA mediated by miR-141. Huh7-HBV1.3 cells were transfected with miR-141 mimic or miR-141 inhibitor. Huh7-HBV1.3 cells were treated with 3-MA or Rap as a negative or positive control group. Results (Fig. 6a-6b) showed that miR-141 mimic significantly increased the expression levels of miR-141 but decreased Sirt1 mRNA, however, it was shown that miR-141 inhibitor significantly decreased the expression 


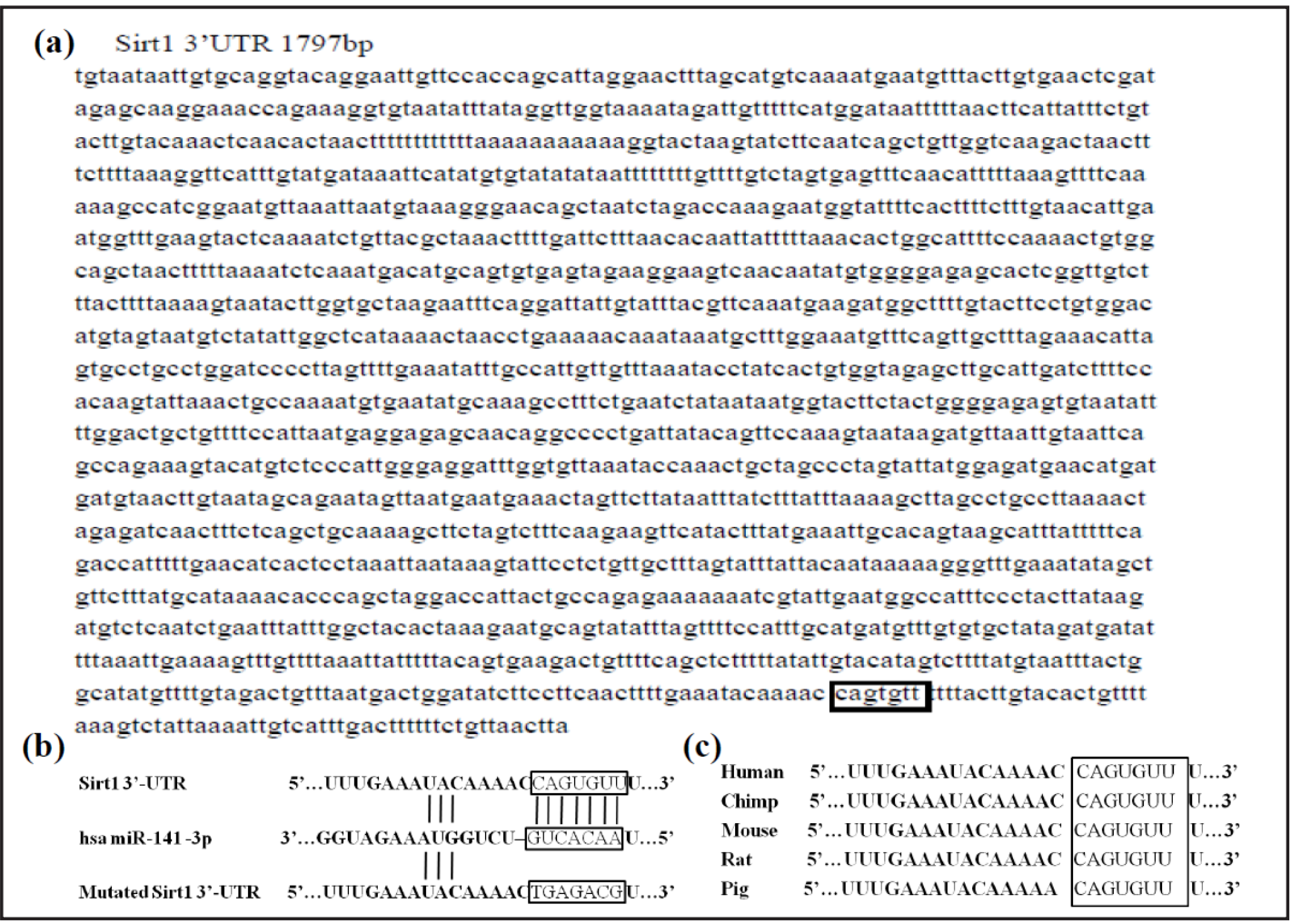

Fig. 4. The Sirt1 3'-UTR sequences for miR-141 binding site and its mutated sequence. The Sirt1 3'-UTR contains one seed match site for miR-141(a). (b) The Sirt1 3'-UTR sequences were mutated to generated the sequences that could not bind to miR-141-3p (Mutated Sirt1 3'-UTR). (c) Alignment details of miR-141-3p on the Sirt1 3'-UTR. Schematic of seed sequences in box match between miR-141-3p and the Sirt1 3'UTR. The miR-141-3p seed match is conserved within many kinds of species including human, chimp, mouse, rat, pig, and so on.

levels of miR-141 but increased Sirt1 mRNA. These results above demonstrated that miR141 could also regulate the expression levels of Sirt1 mRNA.

miR-141 inhibits autophagic activity and HBV replication through Sirt1

SIRT1 regulates the autophagy- lysosome pathway by deacetylation of the major regulators of autophagy including ATG8/LC3. To test the hypothesis that miR-141 inhibits autophagy mediated by Sirt1, we evaluated the autophagic activity in HeLa/GFP-LC3 cells transiently transfected with pSirt1 using fluorescence microscopy to test the number of LC3 puncta.

Fluorescence microscopy results (Fig. $7 \mathrm{a}-7 \mathrm{~d}$ ) showed that transient transfection with pSirt1 activated autophagy in HeLa/GFP-LC3, as suggested by the increased percentage of cells with GFP-LC3 punctate.

Further, results confirmed that Sirt1 promoted HBV replication and expression (Fig. 8a-8c). To further test the hypothesis that miR-141 inhibits autophagy mediated by Sirt1, we evaluated the autophagic activity in Huh7-HBV1.3 cells transiently co-transfected with pSirt1 or Vector and miR-141 mimic or MMNC. Western blot was used to determine the amount of Sirt1, the ratios of LC3-II/LC3-I and p62. Results (Fig. 9a-9d) showed that miR141 mimic or 3-MA significantly reduced the expression levels of Sirt1, the ratios of LC3-II/ LC3-I and p62, as compared to the pSirt1 and the pSirt1 plus MMNC groups. These results validated the hypothesis that miR-141 inhibits autophagy mediated by Sirt1. 


\section{Cellular Physiology Cell Physiol Biochem 2017;41:310-322 and Biochemistry \begin{tabular}{c|c} 
DOI: 10.1159/000456162 \\
Published onnine: anuary 30, 2016 & $\begin{array}{l}\text { O } 2017 \text { The Author(s). Published by S. Karger AG, Basel } \\
\text { www.karger.com/cpb }\end{array}$
\end{tabular} \\ Yang et al.: Inhibition of HBV Replication by MicroRNA-141-Sirt1-Autophagy}

Fig. 5. miR-141 mimic represses the expression of a luciferase reporter containing Sirt1 3 '-UTR. (a) Schematic representation of pGV306-Sirt1 3'-UTR, with the firefly luciferase (Firefly-Luc) reporter construction which was utilized. (b) miR-141 mimic inhibited the Firefly-Luc activities of pGV306-Sirt1 3'-UTR reporter. HepG2.2.15 cells were co-transfected with 200ng pGV306-Sirt1, 200ng pCON172 and with $20 \mathrm{nM}$ miR-141 mimic or miR-141 mimic negative control (MMNC) or transfection reagent (Mock) by Lipofectamine 2000. After 48h transfection, the luciferase reporter activities were assessed. As indicated in Materials and Methods, Firefly-Luc activities were normalized to renilla luciferase activities. Values for cells in Mock group were set equal to 1. (c) Compared to the wild- type Sirt1 sequence included in pGV306 (pSirt1-WT), 3'-UTR luciferase assay showed that miR-141-3p mimic could not reduce expression of the luciferase reporter in pGV306

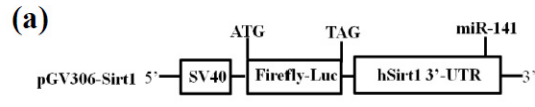

(b)

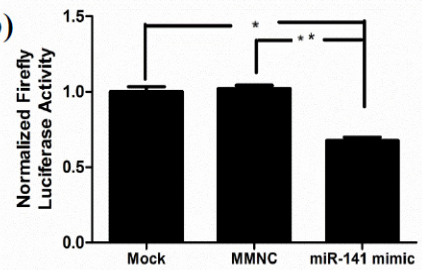

(c)

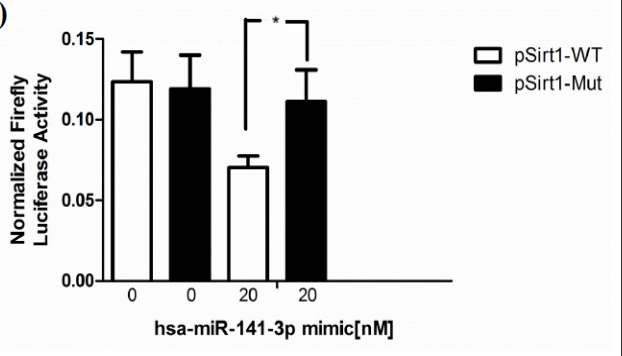
with mutated Sirt1 3'-UTR sequences (pSirt1-Mut). All the results represent the mean \pm SEM data from three independent experiments. ${ }^{*}, P<0.05,{ }^{* *}, P<0.01$.

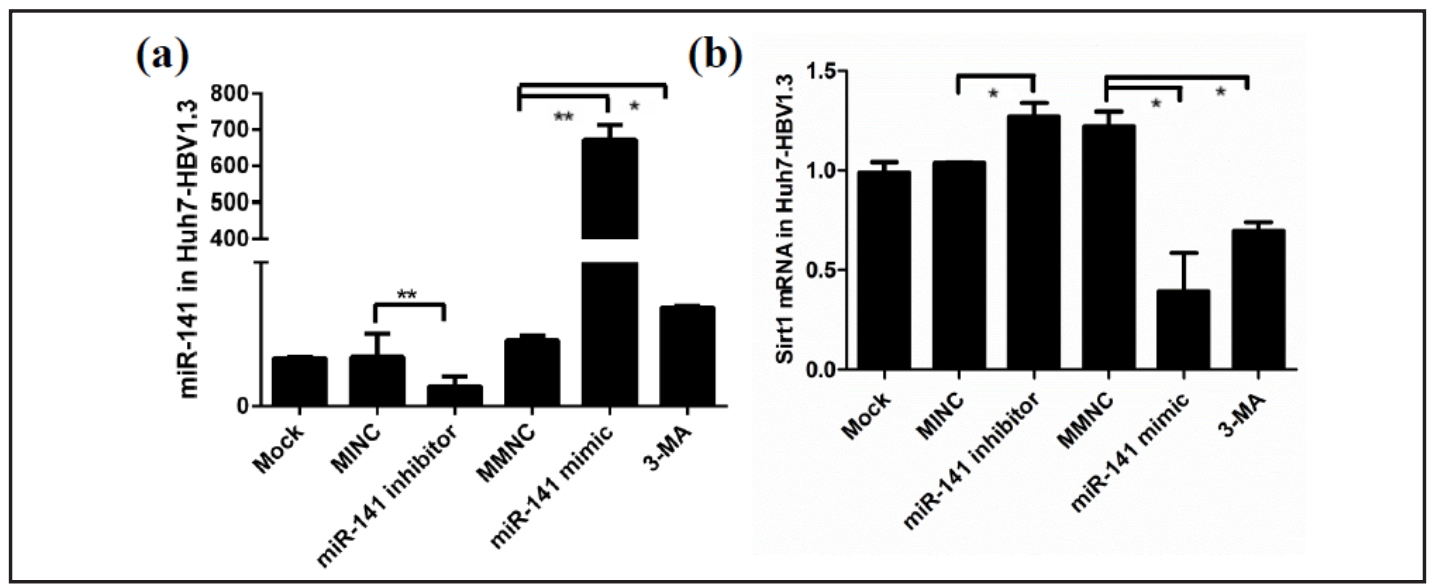

Fig. 6. miR-141 mimic or inhibitor regulates miR-141 and Sirt1 mRNA expression in pHBV1.3 vector transfected Huh7 cells. Huh7-HBV1.3 were transfected with miR-141 mimic or miR-141 inhibitor. pHBV1.3-transfected cells (Huh7-HBV1.3) transfected with miR-141 mimic negative control (MMNC) or miR-141 inhibitor negative control (MINC) or transfection reagent (Mock) was used as a negative control. After 48h, the expression levels of miR-141 (a) and Sirt1 (b) were detected by qPCR. Quantification images were shown above. All the results represent the mean \pm SEM data from three independent experiments. $*, P<0.01, * *, P<0.05$.

\section{Discussion}

Autophagy has been demonstrated to play an important role in HBV replication and pathogenesis. Although it has been reported that the steps in the autophagy pathway are enhanced or subverted by HBV. In Huh7 human hepatoma cells transfected with pEGFP-LC3, the fusion protein of GFP and LC3 is diffusely localized in the cytosol. During autophagy in pHBV1.3-transfected cells (Huh7-HBV1.3), it is localized to autophagosomes. However, the underlying molecular mechanisms remain to be fully demonstrated. Here we reported a novel role of miR-141 in inhibiting autophagy and HBV replication inhibition by targeting Sirt1, which may provide further evidence for host-HBV interaction. 
(a)

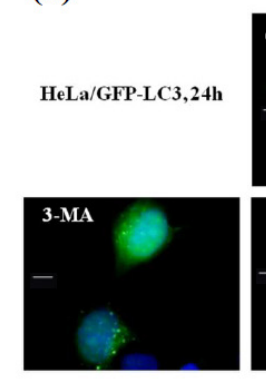

(c)

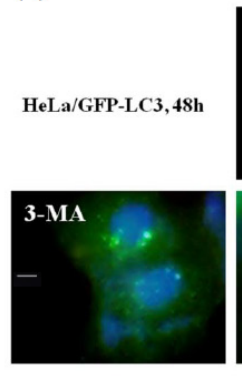

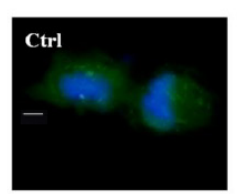
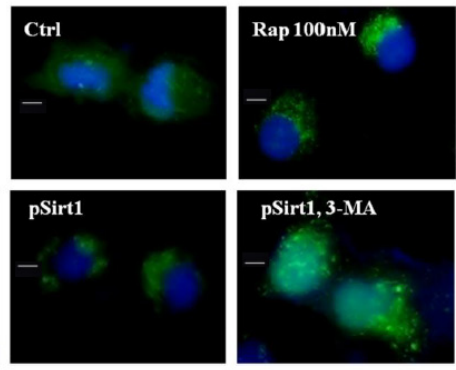

(b)

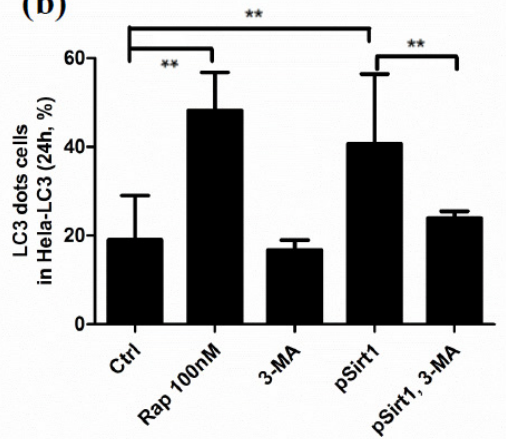

(d)

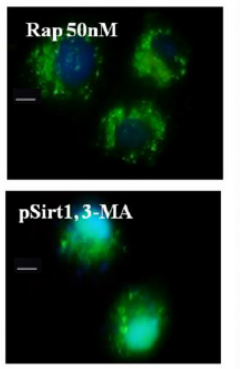

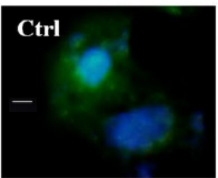

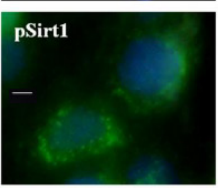

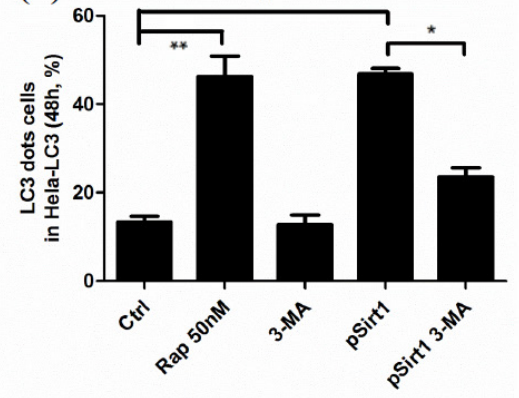

Fig. 7. pSirt1 induces autophagosome in HeLa/GFP-LC3 cells. (a) HeLa/GFP-LC3 cells were transfected with pSirt1, and after $22 \mathrm{~h}$ they were treated or left untreated with 10mM 3-MA for 2h. The GFP-LC3 dots were visualized with a confocal fluorescence microscope. The representative images are shown above. (b) Determination of the frequency of HeLa/GFP-LC3 cells displaying GFP-LC3 dots was performed as described in Materials and Methods. (c) HeLa/GFP-LC3 cells were transfected with pSirt1. After transfection for 46h, the transfected cells were left untreated or were treated with 10mM 3-MA for 2h. (d) Determination of the frequency of HeLa/GFP-LC3 cells displaying GFP-LC3 dots was performed as described in Materials and Methods. Cells treated with $100 \mathrm{nM}$ or 50nM rapamycin (Rap) were used as a positive control. Cells treated with $10 \mathrm{mM} 3-\mathrm{MA}$ were used as a negative control. Scale size: $10 \mu \mathrm{m}$. All the results represent the mean data from three independent experiments. ${ }^{*}, \mathrm{P}<0.01,{ }^{* *}, \mathrm{P}<0.05$.
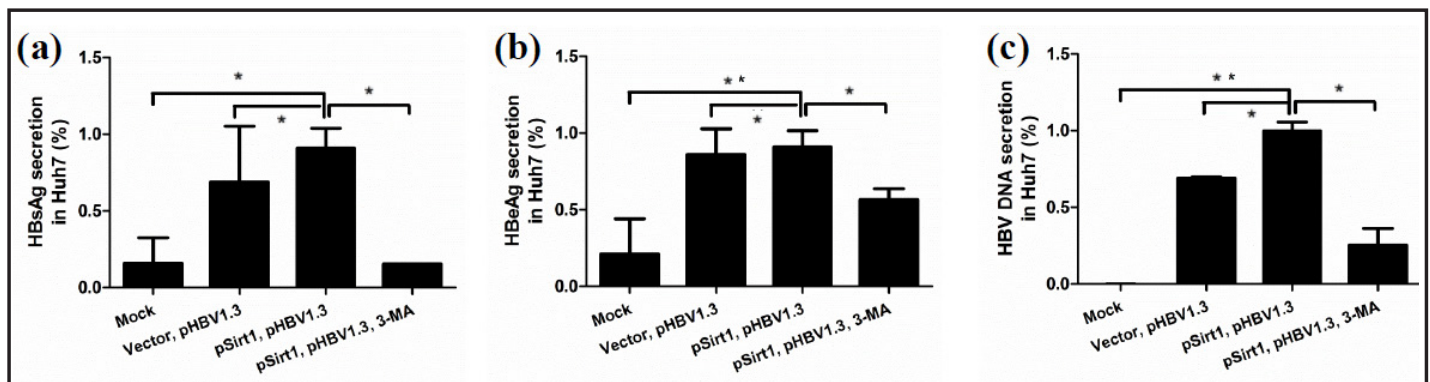

Fig. 8. Sirt1 promotes HBV replication and expression. Co-transfection of Huh7 with pHBV1.3 and pSirt1 was to study HBV replication promotion of Sirt1, and the same group treated with 3-MA was to study the autophagy inhibition on the effect of HBV replication. The vector transfection (Vector, pHBV1.3) and transfection reagent groups (Mock) were used as negative controls. The culture supernatant $48 \mathrm{~h}$ after transfection was harvested. HBsAg (a) and HBeAg (b) of was determined by ELISA assay, and HBV DNA(c) was detected by qPCR. The results represent the mean data from three independent experiments. ${ }^{*}, P<0.05,{ }^{* *}, P<0.01$.

Recent reports have demonstrated that there was an intricate interplay between the host or viral miRNAs and the host response during virus infection including $\operatorname{HBV}[25,26]$. However, among these reports, few focus on the interaction between miRNAs and autophagy. 
(a)

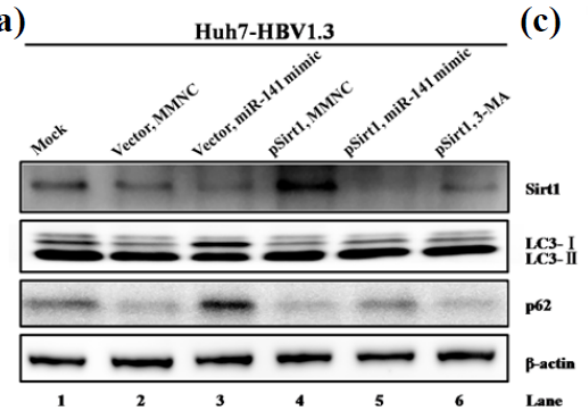

(b)

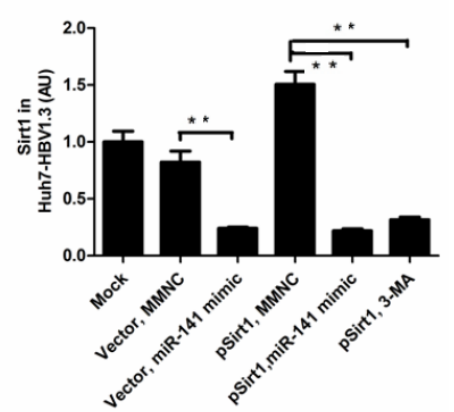

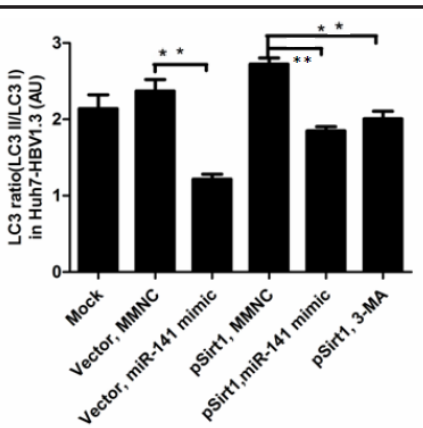

(d)

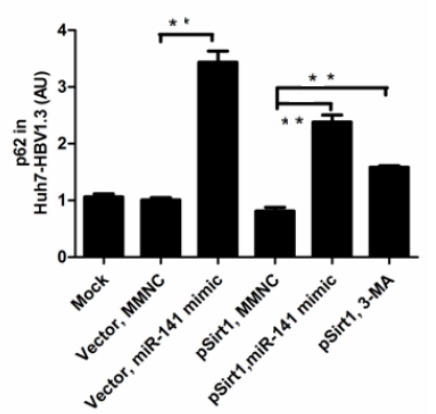

Fig. 9. Sirt1 enhances autophagy affected by miR-141 in Huh7-HBV1.3 cells. pHBV1.3 vector transfected Huh7 cells (Huh7-HBV1.3) were transfected with pSirt1. After 46h, the cells were treated with $10 \mathrm{mM}$ 3-methyladenin (3-MA) or solvent for $2 \mathrm{~h}$ as a negative or positive reagent group. (a-d) The Sirt1, LC3, and p62 levels were detected by Western blot, and the representative images were shown above. The ratios of Sirt1/ $\beta$-actin (b), LC3-II/LC3-I (c) and p62/ $\beta$-actin (d) were shown above. All the results represent the mean \pm SEM data from three independent experiments. ${ }^{* *}, P<0.01$.

It has been reported that miR-141 mimic inhibited HBV expression and replication [25] in HepG2 cells. The present study confirmed the HBV inhibition of miR-141 in Huh7 cells transfected with pHBV1.3 and HepG2.2.15, consistent with the previous study for HBV replication inhibition [25]. It was reported that miR-141 suppressed HBV replication by reducing HBV promoter activities by down-regulating PPARA [25]. Studies have shown that PPAR $\alpha$ activation promotes the autophagy pathway to attenuate the inflammatory response, thus protecting the liver from acute failure [27] and PPAR $\alpha$ inhibits cell apoptosis in vascular adventitial fibroblast partly through the SIRT1-mediated deacetylation of Fox01 [28].

Several markers of autophagy are well characterized, such as microtubule-associated protein light chain 3 (LC3), which undergoes the formation of LC3 II which is converted from LC3-I [29]. p62/SQSTM1 functions an autophagy adaptor and a selective autophagy receptor. p62 undergoes autophagic degradation and degrades at a basal rate $[30,31]$. Notably, our findings showed that miR-141 decreased the accumulation of LC3 puncta and the processing of LC3I to LC3II in HBV1.3 transfected with Huh7 cells, which indicates that miR-141 reduced the autophagic response. Hence, we proposed miR-141 may decrease autophagy response to inhibit HBV replication. Further, the autophagic flux experiment confirmed the autophagy inhibition of miR-141. Autophagy, which was discovered in the liver, is a highly conserved recycling process regulating a number of cell functions including liver metabolism [32,33]. The pathogenesis of various hepatic diseases (including viral hepatitis, hepatocellular carcinoma, and fatty liver) involved the dysregulation of autophagy $[34,35]$. The autophagy can be activated by pharmacological agonists (e.g., rapamycin) [29] or inhibited by pharmacological inhibitors (3-MA) [36]. Emerging evidence has shown that HBV replication appeared to depend on the autophagy machinery [37] in vitro study or in vivo.

Sirt1, which is the most widely studied sirtuin, regulates several biological processes including autophagy and cell metabolism $[38,39]$. Sirt $1 \%$ mice displayed impaired autopha- 


\section{Cellular Physiology Cell Physiol Biochem 2017;41:310-322 \begin{tabular}{ll|l} 
DOI: 10.1159/000456162 & Ond Biochemistry \\
Published onnine: January 30, 2016 & $\begin{array}{l}\text { 2017 The Author(s). Published by S. Karger AG, Basel } \\
\text { www.karger.com/cpb }\end{array}$
\end{tabular} \\ Yang et al.: Inhibition of HBV Replication by MicroRNA-141-Sirt1-Autophagy}

gy, including accumulation of damaged organelles and up-regulation of p62 level [40]. In the present study, we also found that miR-141 down-regulated the expression level of Sirt1, which indicated that miR-141 inhibited autophagy mediated by Sirt1. In addition, we confirmed the autophagic induction of Sirt1 in HeLa/GFP-LC3. The mechanism of Sirt1/autophagy regulation is involved in various kinds of the physiological and pathological process $[41,42]$. The previous study has shown that basal autophagy by deacetylating Atg5, Atg7 and Atg8 was increased by deacetylating Sirt1 [40]. We found that the overexpression of Sirt1 dampened the ability of miR-141 to inhibit autophagic activity in Huh7 transfected with pHBV1.3.

Further, Sirt1 is the target of miR-141 which is predicted by TargetScan, and the data from luciferase assay demonstrated that miR-141 bound to the 3'UTR of Sirt1. Together, our data indicated that Sirt1 was a novel target for miR-141. Studies have demonstrated that miR-141 conducted different functions by repressing various kinds of targets, such as transforming growth factor (TGF)- $\beta 2$ [43], which targets being related to influenza A H5N1. However, none of these studies about miR-141 was involved in modulating autophagic response.

The study was limited by lack of in vivo study, which might weaken the evidence of HBV inhibition by miR-141. The narrow species, tissue tropism of this virus and the lack of reliable laboratory animals susceptible to HBV infection have limited research [44].

Collectively, our study indicated that miR-141 inhibited autophagy by targeting Sirt1, conferring inhibitory effect of HBV replication. The present study demonstrated an important role of miR-141 in autophagy regulation and HBV inhibition, which may provide new light on the development of therapeutic strategy against HBV.

\section{Acknowledgements}

This work was supported by the State S\&T Projects of $12^{\text {th }}$ Five Year (2012ZX10002007) and Chinese National Natural and Science Foundation (No. 81301418).

\section{Disclosure Statement}

The authors confirm that there are no conflicts of interest.

\section{References}

1 Cohen D, Adamovich Y, Reuven N, Shaul Y: Hepatitis b virus activates deoxynucleotide synthesis in nondividing hepatocytes by targeting the $\mathrm{r} 2$ gene. Hepatology 2010;51:1538-1546.

-2 Shen H, Ding F, Wang Z, Sun F, Yu Y, Zhou J, Xu W, Ni J, Wang J, Yang Y: Comparison of telbivudine and entecavir therapy on nephritic function and drug resistance in patients with hepatitis b virus-related compensated cirrhosis. Cell Physiol Biochem 2016;40:370-378.

-3 Chen J, Wang L, Fu Y, Li Y, Bai Y, Luo L, Liao Y: The co-inhibitory pathway and cellular immune imbalance in the progress of hbv infection. Hepatol Int 2014;8:55-63.

4 Han C, Yu L, Liu X, Yu T, Qin W, Liao X, Liu Z, Lu S, Chen Z, Su H, Zhu G, Qin X, Gui Y, Li J, Xiao K, Chen X, Ye $\mathrm{X}$, Peng M, Dong J, Peng T: Atxn7 gene variants and expression predict post-operative clinical outcomes in hepatitis b virus-related hepatocellular carcinoma. Cell Physiol Biochem 2016;39:2427-2438.

5 Silva LM, Jung JU: Modulation of the autophagy pathway by human tumor viruses. Semin Cancer Biol 2013;23:323-328.

6 Petersen J, Thompson AJ, Levrero M: "Aiming for cure in hbv and hdv infection". J Hepatol 2016;65:835848.

7 Mack HI, Munger K: Modulation of autophagy-like processes by tumor viruses. Cells 2012;1:204-247.

> Pratt ZL, Sugden B: How human tumor viruses make use of autophagy. Cells 2012;1:617-630. 


\section{Cellular Physiology Cell Physiol Biochem 2017;41:310-322 \begin{tabular}{l|l|l}
\hline DOI: 10.1159/000456162 30,2016 & $\begin{array}{l}\text { C) } 2017 \text { The Author(s). Published by S. Karger AG, Basel } \\
\text { www.karger.com/cpb }\end{array}$
\end{tabular}}

Yang et al.: Inhibition of HBV Replication by MicroRNA-141-Sirt1-Autophagy

9 Ou X, Lee MR, Huang X, Messina-Graham S, Broxmeyer HE: Sirt1 positively regulates autophagy and mitochondria function in embryonic stem cells under oxidative stress. Stem Cells 2014;32:1183-1194.

10 Ren JH, Tao Y, Zhang ZZ, Chen WX, Cai XF, Chen K, Ko BC, Song CL, Ran LK, Li WY, Huang AL, Chen J: Sirtuin 1 regulates hepatitis b virus transcription and replication by targeting transcription factor ap-1. J Virol 2014;88:2442-2451.

11 Curtil C, Enache LS, Radreau P, Dron AG, Scholtes C, Deloire A, Roche D, Lotteau V, Andre P, Ramiere C: The metabolic sensors fxralpha, pgc-1alpha, and sirt1 cooperatively regulate hepatitis b virus transcription. FASEB J 2014;28:1454-1463.

12 Taganov KD, Boldin MP, Baltimore D: Micrornas and immunity: Tiny players in a big field. Immunity 2007;26:133-137.

13 Kim Y, Lee J, Ryu H: Modulation of autophagy by mirnas. BMB Rep 2015;48:371-372.

14 Guo Y, Chang C, Huang R, Liu B, Bao L, Liu W: Ap1 is essential for generation of autophagosomes from the trans-golgi network. J Cell Sci 2012;125:1706-1715.

15 Yang Z, Kahn BB, Shi H, Xue BZ: Macrophage alpha1 amp-activated protein kinase (alpha1ampk) antagonizes fatty acid-induced inflammation through sirt1. J Biol Chem 2010;285:19051-19059.

16 Guidotti LG, Matzke B, Schaller H, Chisari FV: High-level hepatitis b virus replication in transgenic mice. J Virol 1995;69:6158-6169.

17 Gong DY, Chen EQ Huang FJ, Leng XH, Cheng X, Tang H: Role and functional domain of hepatitis b virus x protein in regulating hbv transcription and replication in vitro and in vivo. Viruses 2013;5:1261-1271.

18 Wiegand J, Brosteanu O, Kullig U, Wiese M, Berr F, Maier M, Tillmann HL, Schiefke I: Quantification of hbsag and hbv-DNA during therapy with peginterferon alpha-2b plus lamivudine and peginterferon alpha- $2 \mathrm{~b}$ alone in a german chronic hepatitis b cohort. Z Gastroenterol 2011;49:1463-1469.

19 Yang Y, Ran J, Sun L, Sun X, Luo Y, Yan B, Tala, Liu M, Li D, Zhang L, Bao G, Zhou J: Cyld regulates noscapine activity in acute lymphoblastic leukemia via a microtubule-dependent mechanism. Theranostics 2015;5:656-666.

20 Yang Y, Li S, Yang Q, Shi Y, Zheng M, Liu Y, Chen F, Song G, Xu H, Wan T, He J, Chen Z: Resveratrol reduces the proinflammatory effects and lipopolysaccharide- induced expression of hmgb1 and tlr4 in raw264.7 cells. Cell Physiol Biochem 2014;33:1283-1292.

21 Sridharan V, Tripathi P, Sharma S, Moros EG, Zheng J, Hauer-Jensen M, Boerma M: Roles of sensory nerves in the regulation of radiation-induced structural and functional changes in the heart. Int J Radiat Oncol Biol Phys 2014;88:167-174.

22 Zhou J, Lu S, Yang S, Chen H, Shi H, Miao M, Jiao B: Microrna-127 post-transcriptionally downregulates sept7 and suppresses cell growth in hepatocellular carcinoma cells. Cell Physiol Biochem 2014;33:15371546.

23 Kopp F, Schnoedt M, Haase R, Wagner E, Roidl A, Ogris M: De-targeting by mir-143 decreases unwanted transgene expression in non-tumorigenic cells. Gene Ther 2013;20:1104-1109.

24 Sir D, Tian Y, Chen WL, Ann DK, Yen TS, Ou JH: The early autophagic pathway is activated by hepatitis b virus and required for viral DNA replication. Proc Natl Acad Sci U S A 2010;107:4383-4388.

-25 Hu W, Wang X, Ding X, Li Y, Zhang X, Xie P, Yang J, Wang S: Microrna-141 represses hbv replication by targeting ppara. PLoS One 2012;7:e34165.

-26 Mosca N, Castiello F, Coppola N, Trotta MC, Sagnelli C, Pisaturo M, Sagnelli E, Russo A, Potenza N: Functional interplay between hepatitis b virus x protein and human mir-125a in hbv infection. Biochem Biophys Res Commun 2014;449:141-145.

-27 Jiao M, Ren F, Zhou L, Zhang X, Zhang L, Wen T, Wei L, Wang X, Shi H, Bai L, Zheng S, Zhang J, Chen Y, Han Y, Zhao C, Duan Z: Peroxisome proliferator-activated receptor alpha activation attenuates the inflammatory response to protect the liver from acute failure by promoting the autophagy pathway. Cell Death Dis 2014;5:e1397.

28 Wang WR, Liu EQ Zhang JY, Li YX, Yang XF, He YH, Zhang W, Jing T, Lin R: Activation of ppar alpha by fenofibrate inhibits apoptosis in vascular adventitial fibroblasts partly through sirt1-mediated deacetylation of foxo1. Exp Cell Res 2015;338:54-63.

29 Delgado M, Singh S, De Haro S, Master S, Ponpuak M, Dinkins C, Ornatowski W, Vergne I, Deretic V: Autophagy and pattern recognition receptors in innate immunity. Immunol Rev 2009;227:189-202.

30 Katsuragi Y, Ichimura Y, Komatsu M: P62/sqstm1 functions as a signaling hub and an autophagy adaptor. FEBS J 2015;282:4672-4678. 


\section{Cellular Physiology Cell Physiol Biochem 2017;41:310-322

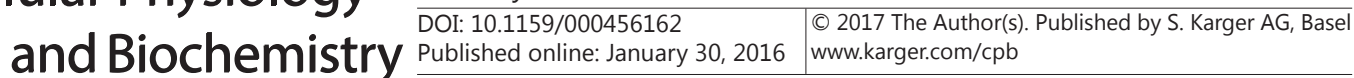 \\ Yang et al.: Inhibition of HBV Replication by MicroRNA-141-Sirt1-Autophagy}

-31 Larsen KB, Lamark T, Overvatn A, Harneshaug I, Johansen T, Bjorkoy G: A reporter cell system to monitor autophagy based on p62/sqstm1. Autophagy 2010;6:784-793.

-32 Schneider JL, Cuervo AM: Liver autophagy: Much more than just taking out the trash. Nat Rev Gastroenterol Hepatol 2014;11:187-200.

33 Madrigal-Matute J, Cuervo AM: Regulation of liver metabolism by autophagy. Gastroenterology 2016;150:328-329.

-34 Ni HM, Williams JA, Yang H, Shi YH, Fan J, Ding WX: Targeting autophagy for the treatment of liver diseases. Pharmacol Res 2012;66:463-474.

-35 Czaja MJ, Ding WX, Donohue TM, Jr., Friedman SL, Kim JS, Komatsu M, Lemasters JJ, Lemoine A, Lin JD, Ou JH, Perlmutter DH, Randall G, Ray RB, Tsung A, Yin XM: Functions of autophagy in normal and diseased liver. Autophagy 2013;9:1131-1158.

-36 Li J, Liu Y, Wang Z, Liu K, Wang Y, Liu J, Ding H, Yuan Z: Subversion of cellular autophagy machinery by hepatitis b virus for viral envelopment. J Virol 2011;85:6319-6333.

37 Tian Y, Sir D, Kuo CF, Ann DK, Ou JH: Autophagy required for hepatitis b virus replication in transgenic mice. J Virol 2011;85:13453-13456.

-38 Michan S, Sinclair D: Sirtuins in mammals: Insights into their biological function. Biochem J 2007;404:113.

-39 Chen J, Zhang B, Wong N, Lo AW, To KF, Chan AW, Ng MH, Ho CY, Cheng SH, Lai PB, Yu J, Ng HK, Ling MT, Huang AL, Cai XF, Ko BC: Sirtuin 1 is upregulated in a subset of hepatocellular carcinomas where it is essential for telomere maintenance and tumor cell growth. Cancer Res 2011;71:4138-4149.

40 Lee IH, Cao L, Mostoslavsky R, Lombard DB, Liu J, Bruns NE, Tsokos M, Alt FW, Finkel T: A role for the naddependent deacetylase sirt1 in the regulation of autophagy. Proc Natl Acad Sci U S A 2008;105:3374-3379.

41 Sun T, Li X, Zhang P, Chen WD, Zhang HL, Li DD, Deng R, Qian XJ, Jiao L, Ji J, Li YT, Wu RY, Yu Y, Feng GK, Zhu XF: Acetylation of beclin 1 inhibits autophagosome maturation and promotes tumour growth. Nat Commun 2015;6:7215.

42 Liu J, Bi X, Chen T, Zhang Q Wang SX, Chiu JJ, Liu GS, Zhang Y, Bu P, Jiang F: Shear stress regulates endothelial cell autophagy via redox regulation and sirt1 expression. Cell Death Dis 2015;6:e1827.

43 Lam WY, Yeung AC, Ngai KL, Li MS, To KF, Tsui SK, Chan PK: Effect of avian influenza a h5n1 infection on the expression of microrna-141 in human respiratory epithelial cells. BMC Microbiol 2013;13:104.

44 Banaudha K, Kaliszewski M, Korolnek T, Florea L, Yeung ML, Jeang KT, Kumar A: Microrna silencing of tumor suppressor dlc-1 promotes efficient hepatitis c virus replication in primary human hepatocytes. Hepatology 2011;53:53-61. 